\title{
CONTINUOUS APPROXIMATION OF QUASIPLURISUBHARMONIC FUNCTIONS
}

\author{
PHILIPPE EYSSIDIEUX, VINCENT GUEDJ, AHMED ZERIAHI
}

\begin{abstract}
Let $X$ be a compact Kähler manifold and $\theta$ a smooth closed $(1,1)$-real form representing a big cohomology class $\alpha \in H^{1,1}(X, \mathbb{R})$. The purpose of this note is to show, using pluripotential and viscosity techniques, that any $\theta$-plurisubharmonic function $\varphi$ can be approximated from above by a decreasing sequence of continuous $\theta$-plurisubharmonic functions with minimal singularities, assuming that there exists a single such function.
\end{abstract}

Dedicated to D.H.Phong on the occasion of his 60th birthday

\section{INTRODUCTION}

Let $X$ be a compact Kähler manifold and $\alpha \in H^{1,1}(X, \mathbb{R})$ be a big cohomology class. Recall that a cohomology class $\alpha \in H^{1,1}(X, \mathbb{R})$ is big if it contains a Kähler current, i.e. a positive closed current which dominates a Kähler form.

Fix $\theta$ a smooth closed real $(1,1)$-form representing $\alpha$. We denote by $P S H(X, \theta)$ the set of all $\theta$-plurisubharmonic functions, i.e. those functions $\varphi: X \rightarrow \mathbb{R} \cup\{-\infty\}$ which can be written locally as the sum of a smooth and a plurisubharmonic function and such that the current $\theta+d d^{c} \varphi$ is a closed positive current, i.e.: $\theta+d d^{c} \varphi \geq 0$ in the sense of currents. It follows from the $\partial \bar{\partial}$-lemma that any closed positive current $T$ in $\alpha$ can be written as $T=\theta+d d^{c} \varphi$ for some $\varphi \in P S H(X, \theta)$. We use the standard normalization

$$
d=\partial+\bar{\partial}, \quad d^{c}:=\frac{1}{2 i \pi}(\partial-\bar{\partial}) \text { so that } d d^{c}=\frac{i}{\pi} \partial \bar{\partial} .
$$

In general Kähler currents are too singular, so one usually prefers to work with positive currents in $\alpha$ having minimal singularities. A positive current $T=\theta+d d^{c} \varphi \in \alpha$ (resp. a $\theta$-plurisubharmonic function $\varphi$ ) has minimal singularities if for every other positive current $S=\theta+d d^{c} \psi \in \alpha$, there exists $C \in \mathbb{R}$ such that $\psi \leq \varphi+C$ on $X$. The function

$$
V_{\theta}:=\sup \left\{v \mid v \in P S H(X, \theta) \text { and } \sup _{X} v \leq 0\right\}
$$

Date: April 11, 2018

The authors are partially supported by the ANR project MACK. 
is an example of $\theta$-psh function with minimal singularities. It satisfies $\sup _{X} V_{\theta}=0$. We let

$$
P(\alpha):=\left\{x \in X \mid V_{\theta}(x)=-\infty\right\} \text { and } N B(\alpha):=\left\{x \in X \mid V_{\theta} \notin L_{l o c}^{\infty}(\{x\})\right\}
$$

denote respectively the polar locus and the non bounded locus of $\alpha$. The definitions clearly do not depend on the choice of $\theta$ and coincide with the polar (resp. non bounded locus) of any $\theta$-psh function with minimal singularities.

The purpose of this note is to show that if a big cohomology class contains one current having minimal singularities and exponentially continuous potentials, then there is actually plenty of such currents:

THEOREM. Let $X$ be a compact Kähler manifold and let $\alpha \in H^{1,1}(X, \mathbb{R})$ be a big cohomology class such that the polar locus $P(\alpha)$ coincides with the non-bounded locus $N B(\alpha)$.

Fix $\theta \in \alpha$ a smooth representative and $T=\theta+d d^{c} \varphi$ a positive current in $\alpha$, where $\varphi \in P S H(X, \theta)$. Then there exists $\varphi_{j} \in P S H(X, \theta)$ a sequence of exponentially continuous $\theta$-plurisubharmonic functions which have minimal singularities and decrease towards $\varphi$.

We say here that a $\theta$-psh function is exponentially continuous iff $e^{\varphi}: X \rightarrow$ $\mathbb{R}$ is continuous. Observe that if there exists one exponentially continuous $\theta$-psh function with minimal singularities, then $P(\alpha)=N B(\alpha)$.

The technical condition $P(\alpha)=N B(\alpha)$ is thus necessary. It is obviously satisfied when $\alpha$ is semi-positive, or even bounded (i.e. there exists a positive closed current in $\alpha$ with bounded potentials, a condition that has become important in complex dynamics recently, see [DG09]), since $P(\alpha)=N B(\alpha)=\emptyset$ in this case. A more subtle example of a big and nef class $\alpha$ with $P(\alpha)=N B(\alpha) \neq \emptyset$ has been given in [BEGZ10, Example 5.4].

It is easy to construct $\theta$-plurisubharmonic functions $\psi$ with $P(\psi) \subsetneq$ $N B(\psi)$, however we do not know of a single example of a big class $\alpha$ for which $P(\alpha)$ is strictly smaller than $N B(\alpha)$.

Despite the relative modesty of its conclusion, this result relies on three important tools:

-the regularization techniques of Demailly as used in BD12,

-the resolution of degenerate complex Monge-Ampère equations in big cohomology classes, as developed in BEGZ10,

-and the viscosity approach to complex Monge-Ampère equations [EGZ11].

The latter was developed in the case where $\alpha$ is both big and semi-positive, hence we need here to extend this technique so as to cover the general setting of big classes. We stress that the global viscosity comparison principle holds for a big cohomology class $\alpha$ if and only if $P(\alpha)=N B(\alpha)$ (Theorem 3.4).

Our approximation result is new even when $\alpha$ is both big and semipositive. Let us stress that continuous $\theta$-plurisubharmonic functions are 
easy to regularize by using Richberg's technique [R68. As a consequence we obtain the following:

COROLLARY. Let $\left(V, \omega_{V}\right)$ be a compact normal Kähler space and let $\varphi$ be a $\omega_{V}$-plurisubharmonic function on $V$. Then there exists a sequence $\left(\varphi_{j}\right)$ of smooth $\omega_{V}$-plurisubharmonic functions which decrease towards $\varphi$.

When $\omega_{V}$ is a Hodge form, this regularization can be seen as a consequence of the extension result of CGZ13.

Approximation from above by regular objects is of central use in the theory of complex Monge-Ampère operators, as the latter are continuous along (and even defined through) such monotone sequences [BT82, while they are not continuous with respect to the weaker $L^{1}$-topology Ceg83.

Plan of the note. We first establish our main result when the underlying cohomology class is also semi-positive (section 2), as the viscosity technology is already available EGZ11; the corollary follows then easily by using Richberg's regularization result. We then (section 3) adapt the techniques of EGZ11] to the general context of big cohomology classes. The technical condition $P(\alpha)=N B(\alpha)$ naturally shows up as it is necessary for the global viscosity comparison principle to hold. We finally (section 44) use recent stability estimates for big cohomology classes GZ12 to obtain continuous solutions of slightly more general Monge-Ampère equations, which allow us to prove our main result.

Dédicace. C'est un plaisir de contribuer à ce volume en l'honneur de Duong Hong Phong, dont nous apprécions la générosité, la vision et le bon goût, tant mathématique que gastronomique!

\section{The CASE OF SEMI-POSITIVE CLASSES}

We fix once and for all $\left(X, \omega_{X}\right)$ a compact Kähler manifold of complex dimension $n, \alpha \in H^{1,1}(X, \mathbb{R})$ a big cohomology class and $\theta$ a smooth closed $(1,1)$ form representing $\alpha$.

2.1. Minimal vs analytic singularities. Recall that $\alpha$ is semi-positive if $\theta$ can be chosen to be a semi-positive form. In this case a $\theta$-psh function has minimal singularities if and only if its is bounded. The easiest example of $\theta$-psh functions with minimal singularities are constant functions which are indeed $\theta$-psh iff $\theta$ is semipositive.

For more general $\alpha, \theta$-psh function with minimal singularities can be constructed as enveloppes, e.g.:

$$
V_{\theta}:=\sup \left\{v \mid v \in P S H(X, \theta) \text { and } \sup _{X} v \leq 0\right\} .
$$

Note that if $V \in P S H(X, \theta)$ is another function with minimal singularities, then $V-V_{\theta}$ is globally bounded on $X$. Also if $\theta^{\prime}=\theta+d d^{c} \rho$ is another smooth form representing $\alpha$, then $\operatorname{PSH}\left(X, \theta^{\prime}\right)=\operatorname{PSH}(X, \theta)-\rho$ where $\rho \in \mathcal{C}^{\infty}(X, \mathbb{R})$ hence $V_{\theta}-V_{\theta^{\prime}}$ is also globally bounded on $X$. 
Definition 2.1. The polar locus of $\alpha$ is

$$
P(\alpha):=\left\{x \in X \mid V_{\theta}(x)=-\infty\right\} .
$$

The non-bounded locus of $\alpha$ is

$$
N B(\alpha):=\left\{x \mid V_{\theta} \notin L_{l o c}^{\infty}(\{x\})\right\}
$$

The observations above show that these definitions only depend on $\alpha$. Clearly $P(\alpha) \subset N B(\alpha)$ and $N B(\alpha)$ is closed. We shall assume in the sequel that $P(\alpha)=N B(\alpha)$ which, as will turn out, is equivalent to saying that there exists one exponentially continuous $\theta$-psh function with minimal singularities.

By definition $\alpha$ is big if it contains a Kähler current, i.e. there is a (singular) positive current $T \in \alpha$ and $\varepsilon>0$ such that $T \geq \varepsilon \omega_{X}$. It follows from the regularization techniques of Demailly (see [Dem92]) that one can further assume that $T$ has analytic singularities:

Definition 2.2. A positive closed current $T$ has analytic singularities if it can be locally written $T=d d^{c} u$, with

$$
u=\frac{c}{2} \log \left[\sum_{j=1}^{s}\left|f_{j}\right|^{2}\right]+v,
$$

where $c>0, v$ is smooth and the $f_{j}$ 's are holomorphic functions.

We let $\operatorname{Amp}(\alpha)$ denote the ample locus of $\alpha$, i.e. the Zariski open subset of all points $x \in X$ for which there exists a Kähler current in $\alpha$ with analytic singularities which is smooth in a neighborhood of $x$.

It follows from the work of Boucksom [Bou04] that one can find a single Kähler current $T_{0}=\theta+d d^{c} \psi_{0}$ with analytic singularities in $\alpha$ such that

$$
\operatorname{Amp}(\alpha)=\mathrm{X} \backslash \operatorname{Sing} \mathrm{T}_{0}
$$

In the sequel we fix such a Kähler current $T_{0}$ and assume for simplicity that

$$
T_{0} \geq \omega_{X}
$$

Observe that $\psi_{0}$ is exponentially continuous, however $\psi_{0}$ does not have minimal singularities unless $\alpha$ is Kähler (see [Bou04]).

Bounded vs continuous approximations. Fix $\varphi \in P S H(X, \theta)$ a $\theta$-psh function. It is easy to approximate $\varphi$ from above by a decreasing sequence of $\theta$-psh functions with minimal singularities. Indeed we can set

$$
\varphi_{j}:=\max \left(\varphi, V_{\theta}-j\right) \in P S H(X, \theta) .
$$

The latter have minimal singularities and decrease to $\varphi$ as $j \nearrow+\infty$. This construction needs however to be refined to get exponentially continuous $\theta$-psh approximations with minimal singularities. The mere existence of exponentially continuous $\theta$-psh functions $\psi$ with minimal singularities is actually not obvious. 
2.2. Continuous approximations in the semi-positive case. We show here our main result in the simpler case when $\alpha$ is both big and semi-positive.

Theorem 2.3. Assume $\alpha \in H^{1,1}(X, \mathbb{R})$ is big and semi-positive and let $\varphi \in P S H(X, \theta)$ be a $\theta$-plurisubharmonic function.

Then there exists a sequence of continuous $\theta$-plurisubharmonic functions which decrease towards $\varphi$.

Proof. Fix $h_{j}$ a sequence of smooth functions decreasing to $\varphi$ (recall that $\varphi$ is upper semi-continuous) and set

$$
\varphi_{j}=P\left(h_{j}\right):=\sup \left\{u \mid u \in P S H(X, \theta) \text { and } u \leq h_{j}\right\} .
$$

Observe that $\varphi_{j} \in P S H(X, \theta)$ and $\varphi_{j} \leq h_{j}$ hence

$$
\varphi \leq \varphi_{j+1} \leq \varphi_{j}
$$

We claim that $\varphi=\lim \searrow \varphi_{j}$. Indeed set $\psi:=\lim \searrow \varphi_{j} \geq \varphi$. Then $\psi \leq h_{j}$ for all $j$ and $\psi \in P S H(X, \theta)$, hence $\psi \leq \varphi$, so that $\psi=\varphi$ as claimed.

It thus suffices to check that $\varphi_{j}$ is continuous. It follows from the work of Berman and Demailly BD12 that $\varphi_{j}$ has locally bounded Laplacian on the ample locus $\operatorname{Amp}(\alpha)$ of $\alpha$, with

$$
\left(\theta+d d^{c} \varphi_{j}\right)^{n}=\mathbf{1}_{\left\{P\left(h_{j}\right)=h_{j}\right\}}\left(\theta+d d^{c} h_{j}\right)^{n} \text { in } \operatorname{Amp}(\alpha) .
$$

The measure on the right hand side is absolutely continuous with respect to Lebesgue measure, with bounded density. It therefore follows from [EGZ11, Theorem C] that $\varphi_{j}=P\left(h_{j}\right)$ is continuous.

Remark 2.4. Observe that a key point in the proof above is that if $h$ is a smooth function on $X$, then its $\theta$-plurisubharmonic projection $P(h)$ is a continuous $\theta$-plurisubharmonic function with minimal singularities.

Although the proof is quite short in appearance, it uses several important tools: Demailly's regularization technique (which is heavily used in [BD12]), and the viscosity approach for degenerate complex Monge-Ampère equations developed in EGZ11.

The proof of our main theorem follows exactly the same lines: the result of Berman-Demailly applies for general big classes, while [BEGZ10] produces solutions of complex Monge-Ampère equations with minimal singularities in big cohomology classes. It thus remains to extend the viscosity approach of EGZ11 to the setting of big cohomology classes, which is the contents of the next section.

Since Richberg's regularization technique [R68] applies in a singular setting, we obtain the following interesting consequence:

Corollary 2.5. Let $\left(V, \omega_{V}\right)$ be a compact normal Kähler space and let $\varphi$ be $a \omega_{V}$-plurisubharmonic function on $V$. Then there exists a sequence $\left(\varphi_{j}\right)$ of smooth $\omega_{V}$-plurisubharmonic functions which decrease towards $\varphi$. 
Proof. Fix $\varphi \in P S H\left(X, \omega_{V}\right)$. We can assume without loss of generality that $\varphi<0$ on $V$. Let $\pi: X \rightarrow V$ be a desingularization of $V$ and set $\theta:=\pi^{*} \omega_{V}$. Then $\psi:=\varphi \circ \pi \in P S H(X, \theta)$.

Since $\pi^{*}\left\{\omega_{V}\right\} \in H^{1,1}(X, \mathbb{R})$ is big, it follows from our previous result that we can find continuous $\theta$-psh functions $\psi_{j}<0$ which decrease towards $\psi$ on $X$. Since $\pi$ has connected fibers, one easily checks that

$$
P S H(X, \theta)=\pi^{*} P S H\left(V, \omega_{V}\right),
$$

in particular there exists $\varphi_{j} \in P S H\left(V, \omega_{V}\right) \cap \mathcal{C}^{0}(V)$ such that $\psi_{j}:=\varphi_{j} \circ \pi$, with $\varphi_{j}$ decreasing to $\varphi$.

We can now invoke Richberg's regularization result [R68] (see also [Dem92]): using local convolutions and patching, one can find smooth functions $\left(\varphi_{j, k}\right)$ on $V$ which decrease to $\varphi_{j}$ as $k \rightarrow+\infty$ and such that $\varphi_{j, k} \in \operatorname{PSH}(V,(1+$ $\left.\left.\varepsilon_{k}\right) \omega_{V}\right)$ with $\varepsilon_{k} \searrow 0$. We can also assume that $\varphi_{j, k}<0$ on $V$. Set finally

$$
u_{j}:=\frac{1}{\left(1+\varepsilon_{j}\right)} \varphi_{j, j} \in P S H\left(V, \omega_{V}\right) \cap \mathcal{C}^{\infty}(V) .
$$

We let the reader check that $\left(u_{j}\right)$ still decreases to $\varphi$.

Remark 2.6. When $\omega_{V}$ has integer class, i.e. when it represents the first Chern class of an ample line bundle on $V$, the above result was obtained in [CGZ13] as a consequence of an extension result of $\omega_{V}$-psh functions.

\section{Viscosity APPROACH IN A BIG SETting}

We set here the basic frame for the viscosity approach to the equation

$$
\left(\theta+d d^{c} \varphi\right)^{n}=e^{\varepsilon \varphi} v
$$

where $v$ is a volume form with nonnegative continuous density and $\varepsilon>0$ is a real parameter.

3.1. Viscosity sub/super-solutions for big cohomology classes. To fit in with the viscosity point of view, we rewrite the Monge-Ampère equation as

$$
e^{\varepsilon \varphi} v-\left(\theta+d d^{c} \varphi\right)^{n}=0
$$

Let $x \in X$. If $\kappa \in \Lambda^{1,1} T_{x} X$ we define $\kappa_{+}^{n}$ to be $\kappa^{n}$ if $\kappa \geq 0$ and 0 otherwise. For a technical reason, we will also consider a slight variant of $\left(D M A_{v}^{\varepsilon}\right)$,

$\left(D M A_{v}^{\varepsilon}\right)_{+} \quad e^{\varepsilon \varphi} v-\left(\theta+d d^{c} \varphi\right)_{+}^{n}=0$

If $\varphi_{x}^{(2)}$ is the 2-jet at $x \in X$ of a $\mathcal{C}^{2}$ real valued function $\varphi$ we set

$$
F\left(\varphi_{x}^{(2)}\right)=F_{v}^{\varepsilon}\left(\varphi_{x}\right)= \begin{cases}e^{\varepsilon \varphi(x)} v_{x}-\left(\theta_{x}+d d^{c} \varphi_{x}\right)^{n} & \text { if } \theta+d d^{c} \varphi_{x} \geq 0 \\ +\infty & \text { otherwise. }\end{cases}
$$

Recall the following definition from [CIL92, [EGZ11, Definition 2.3]: 
Definition 3.1. A subsolution of $\left(D M A_{v}^{\varepsilon}\right)$ is an upper semi-continuous function $\varphi: X \rightarrow \mathbb{R} \cup\{-\infty\}$ such that $\varphi \not \equiv-\infty$ and the following property is satisfied: if $x_{0} \in X$ and $q \in \mathcal{C}^{2}$, defined in a neighborhood of $x_{0}$, is such that $\varphi\left(x_{0}\right)=q\left(x_{0}\right)$ and

$$
\varphi-q \text { has a local maximum at } x_{0},
$$

then $F\left(q_{x_{0}}^{(2)}\right) \leq 0$.

We say that $\varphi$ has minimal singularities if there exists $C>0$ such that

$$
V_{\theta}-C \leq \varphi \leq V_{\theta}+C \text { on } X \text {. }
$$

It has been shown in [EGZ11, Corollary 2.6] that a viscosity subsolution $\varphi$ of $\left(D M A_{v}^{\varepsilon}\right)$ is a $\theta$-psh function which satisfies $\left(\theta+d d^{c} \varphi\right)^{n} \geq e^{\varepsilon \varphi} v$ in the pluripotential sense of [BT82, BEGZ10].

We now slightly extend the concept of supersolution, so as to allow a supersolution to take $-\infty$ values:

Definition 3.2. A supersolution of $\left(D M A_{v}^{\varepsilon}\right)$ is a supersolution of $\left(D M A_{v}^{\varepsilon}\right)_{+}$, that is, a function $\varphi: X \rightarrow \mathbb{R} \cup\{ \pm \infty\}$ such that $e^{\varphi}$ is lower semicontinuous, $\varphi \not \equiv+\infty, \varphi \not \equiv-\infty$ and the following property is satisfied: if $x_{0} \in X$ and $q \in \mathcal{C}^{2}$, defined in a neighborhood of $x_{0}$, is such that $\varphi\left(x_{0}\right)=q\left(x_{0}\right)$ and

$$
\varphi-q \text { has a local minimum at } x_{0},
$$

then $F_{+}\left(q_{x_{0}}^{(2)}\right) \geq 0$.

We say that $\varphi$ has minimal singularities if there exists $C>0$ such that

$$
\left(V_{\theta}\right)_{*}-C \leq \varphi \leq\left(V_{\theta}\right)_{*}+C \text { on } X .
$$

Here $\left(V_{\theta}\right)_{*}$ denotes the lower semi-continuous regularization of $V_{\theta}$. It is important to allow $-\infty$ values since we are trying to build a $\theta$-psh viscosity solution of $\left(D M A_{v}^{\varepsilon}\right)$ : in general such a function will be infinite at polar points $x_{0} \in P(\alpha)$. Note that we don't impose any condition at such points.

Definition 3.3. A viscosity solution of $\left(D M A_{v}^{\varepsilon}\right)$ is a function that is both a sub-and a supersolution. In particular a viscosity solution $\varphi$ is automatically an exponentially continuous $\theta$-plurisubharmonic function.

By comparison, a pluripotential solution of $\left(D M A_{v}^{\varepsilon}\right)$ is an usc function $\varphi \in L_{l o c}^{\infty}(\operatorname{Amp}(\alpha)) \cap \operatorname{PSH}(\mathrm{X}, \omega)$ such that

$$
\left(\theta+d d^{c} \varphi\right)_{B T}^{n}=e^{\varepsilon \varphi} v \text { in } \operatorname{Amp}(\alpha)
$$

in the sense of Bedford-Taylor [BT82] (see [BEGZ10] for the slightly more general notion of non-pluripolar products): it follows from BEGZ10 that such a pluripotential solution automatically has minimal singularities, however there is no continuity information, especially at points in $X \backslash \operatorname{Amp}(\alpha)$, as this set is pluripolar hence invisible from the pluripotential point of view. 


\subsection{The big viscosity comparison principle.}

Theorem 3.4. Let $\alpha \in H^{1,1}(X, \mathbb{R})$ be a big cohomology class and assume $\varepsilon>0$ and $v>0$. Let $\varphi$ (resp. $\psi$ ) be a subsolution (resp. supersolution) of $\left(D M A_{v}^{\varepsilon}\right)$ with minimal singularities, then

$$
\varphi \leq \psi \text { in } \operatorname{Amp}(\alpha) \text {. }
$$

Moreover $\varphi \leq \psi$ on $X$ if and only if $P(\alpha)=N B(\alpha)$.

Proof. We can assume $\varepsilon=1$ without loss of generality.

We let $x_{0} \in X$ denote a point that realizes the maximum of $e^{\varphi}-e^{\psi}$ on $X$. If $x_{0} \in P(\alpha)$, then we conclude trivially: $\varphi\left(x_{0}\right)=-\infty$, hence $\max _{X}\left(e^{\varphi}-e^{\psi}\right) \leq 0$.

Assume now $x_{0} \notin \mathrm{NB}(\alpha)$. Then $\varphi$ and $\psi$ are locally bounded near $x_{0}$. Since $N B(\alpha)$ is closed, we can choose complex coordinates $\left(z^{1}, \ldots, z^{n}\right)$ near $x_{0}$ defining a biholomorphism identifying an open neighborhood of $x_{0}$ in $X-\operatorname{NB}(\alpha)$ to the complex ball $B(0,5) \subset \mathbb{C}^{n}$ of radius 5 sending $x_{0}$ to the origin.

We define $h \in \mathcal{C}^{2}(\overline{B(0,5)}, \mathbb{R})$ to be a local potential smooth up to the boundary for $\theta$ and extend it smoothly to $X$. In particular $d d^{c} h=\theta$ and $w_{-}:=\varphi+h$ is a bounded viscosity subsolution of the equation

$$
\left(d d^{c} w\right)^{n}=e^{w} W \text { in } B(0,5)
$$

with $W$ a positive and continuous volume form. On the other hand $w^{+}=$ $\psi+h$ is a bounded viscosity supersolution of the same equation.

Now choose $C>0$ such that $\sup _{x \in \overline{B(0,4)}} \max (|\varphi(x)|,|\psi(x)|) \leq C / 1000$ and $\sup _{x \in \overline{B(0,4)}}|h(x)| \leq C / 10$. With this constant $C>0$, construct as in [EGZ11, p. 1076] a smooth auxiliary function $\varphi_{3}$ on $\overline{B(0,4)}^{2}$. Using the same notations as in [EGZ11, p. 1077], fix $\beta>0$ and consider $\left(x_{\beta}, y_{\beta}\right) \in \overline{B(0,4)}^{2}$ such that:

$$
\begin{aligned}
M_{\beta} & =\sup _{(x, y) \in \overline{B(0,4)}^{2}} w_{-}(x)-w^{+}(y)-\varphi_{3}(x, y)-\frac{\beta}{2} d^{2}(x, y) \\
& =w_{-}\left(x_{\beta}\right)-w^{+}\left(y_{\beta}\right)-\varphi_{3}\left(x_{\beta}, y_{\beta}\right)-\frac{\beta}{2} d^{2}\left(x_{\beta}, y_{\beta}\right) .
\end{aligned}
$$

By construction, $\varphi_{3}$ is big enough outside $B(0,2)^{2}$ to ensure that the sup is achieved at some point $\left(x_{\beta}, y_{\beta}\right) \in B(0,2)^{2}$. Limit points $(x, y)$ of $\left(x_{\beta}, y_{\beta}\right)$ satisfy $x=y$ and the construction forces $\varphi_{3}$ to vanishes to high order at such a limit point. Then, the argument of [EGZ11, p. 1077-1078] based on Ishii's version of the maximum principle (see [CIL92]) applies verbatim to prove that

$$
\limsup _{\beta \rightarrow 0} w^{+}\left(x_{\beta}\right)-w^{-}\left(y_{\beta}\right) \geq 0
$$

and enables us to conclude that $\varphi \leq \psi$.

However, if $x_{0} \in N B(\alpha) \backslash P(\alpha), \varphi\left(x_{0}\right)>-\infty$ since $\phi$ has minimal singularities, while $\psi\left(x_{0}\right)=-\infty$ since $\psi$ has minimal singularities in the sense 
of definition 3.2 and $x_{0} \in N B(\alpha)$ implies that $\left(V_{\theta}\right)_{*}\left(x_{0}\right)=-\infty$. The global comparison principle thus fails if $P(\alpha) \neq N B(\alpha)$.

Let us now justify that in general we do have $\varphi \leq \psi$ on $\operatorname{Amp}(\alpha)$. Let $T_{0}=\theta+d d^{c} \psi_{0}$ be a Kähler current such that $\operatorname{Amp}(\alpha)=\mathrm{X} \backslash \operatorname{Sing}\left(\mathrm{T}_{0}\right), \psi_{0} \leq \psi$ and $\psi_{0} \leq 0$. Fix $\delta>0$ and consider $\varphi_{\delta}:=(1-\delta) \varphi+\delta \psi_{0}+n \log (1-\delta)$. We claim that $\varphi_{\delta}$ is again a subsolution of $\left(D M A_{v}^{\varepsilon}\right)$. Indeed there is nothing to test on $\operatorname{Sing}\left(T_{0}\right)$, while in $\operatorname{Amp}(\alpha)$

$$
\left(\theta+d d^{c} \varphi_{\delta}\right)^{n} \geq(1-\delta)^{n}\left(\theta+d d^{c} \varphi\right)^{n} \geq(1-\delta)^{n} e^{\varphi} v \geq e^{\varphi_{\delta}} v,
$$

as follows easily by interpreting these inequalities in the pluripotential sense (see [EGZ11, Proposition 1.11]).

Let $x_{\delta}$ be a point where the upper semi-continuous function $e^{\varphi_{\delta}}-e^{\psi}$ attains its maximum. If $x_{\delta} \in \operatorname{Sing}\left(T_{0}\right)$, then $e^{\varphi_{\delta}\left(x_{\delta}\right)}=0$ hence

$$
e^{\varphi_{\delta}} \leq e^{\psi} \Rightarrow \varphi_{\delta} \leq \psi \text { on } X \text {. }
$$

If $x_{\delta} \in \operatorname{Amp}(\alpha)$, then both $\varphi_{\delta}$ and $\psi$ are locally bounded near $x_{\delta}$ and the argument above leads to the conclusion that $e^{\varphi_{\delta}\left(x_{\delta}\right)} \leq e^{\psi\left(x_{\delta}\right)}$ hence $\varphi_{\delta} \leq \psi$ on $X$. Letting $\delta$ decrease to zero, we infer that $\varphi \leq \psi$ in $\operatorname{Amp}(\alpha)$.

\subsection{Continuous solutions of big Monge-Ampère equations.}

Theorem 3.5. Let $\alpha \in H^{1,1}(X, \mathbb{R})$ be a big cohomology class and assume $\varepsilon>0$ and $v>0$ is a continuous positive density. Then there exists a unique pluripotential solution $\varphi$ of $\left(D M A_{v}^{\varepsilon}\right)$ on $X$, such that

(1) $\varphi$ is a $\theta$-plurisubharmonic function with minimal singularities,

(2) $\varphi$ is a viscosity solution in $\operatorname{Amp}(\alpha)$ hence continuous there,

(3) Its lower semicontinuous regularisation $\varphi_{*}$ is a viscosity supersolution.

If $P(\alpha)=N B(\alpha)$ then $\varphi$ is a viscosity solution of $\left(D M A_{v}^{\varepsilon}\right)$ on $X$, hence $e^{\varphi}$ is continuous on $X$.

Proof. We can always assume that $\varepsilon=1$. Since the comparison principle holds on $\operatorname{Amp}(\alpha)$, we can use Perron's method by considering the upper enveloppe of subsolutions.

It follows from [BEGZ10] that the equation $\left(D M A_{v}^{1}\right)$ has a pluripotential solution $\phi_{0}$ which is a $\theta$-plurisubharmonic function on $X$ satisfying the equation $\left(\theta+d d^{c} \phi_{0}\right)^{n}=e^{\phi_{0}} v$ weakly on $X$. Since the right hand side is a bounded volume form, it follows from the big version of Kolodziej's uniform estimates that $\varphi_{0}$ has minimal singularities.

Moreover from the definition of a subsolution in the big case and [EGZ11, Corollary 2.6], it follows that $\varphi_{0}$ is a viscosity subsolution to the equation $\left(D M A_{v}^{1}\right)$.

On the other hand, since by BD12, $V_{\theta}$ satisfies the equation $\left(\theta+d d^{c} V_{\theta}\right)^{n}=$ $\mathbf{1}_{\left\{V_{\theta}=0\right\}} \theta^{n}$ in the pluripotential sense and the right hand side is a bounded volume form, it follows that for some constant $C>>1$ the function $\phi_{1}:=$ $V_{\theta}+C$ satisfies the inequality $\left(\theta+d d^{c} \phi_{1}\right)^{n} \leq e^{\phi_{1}} v$ in pluripotential sense. It 
follows therefore from the proof of [EGZ11, Lemma 4.7(1)] that $\psi_{1}:=\left(\phi_{1}\right)_{*}$ is a (viscosity) supersolution to the equation $\left(D M A_{v}^{1}\right)$.

We can now consider the upper envelope of (viscosity) subsolutions,

$$
\varphi:=\sup \left\{\psi ; \psi \text { viscosity subsolution, } \phi_{0} \leq \psi \leq \psi_{1}\right\},
$$

which is a subsolution with minimal singularities to the equation $\left(D M A_{v}^{1}\right)$. Using the bump construction ([CIL92, EGZ11]) we can show that the lower semi-continuous regularization $\varphi_{*}$ of $\varphi$ is a (viscosity) supersolution with minimal singularities to the equation $\left(D M A_{v}^{1}\right)$.

Therefore since the comparison principle holds on $\operatorname{Amp}(\alpha)$, it follows that $\varphi \leq \varphi_{*}$ on $\operatorname{Amp}(\alpha)$, hence $\varphi=\varphi_{*}$ on $\operatorname{Amp}(\alpha)$ is a viscosity solution to the equation $\left(D M A_{v}^{1}\right)$ on $\operatorname{Amp}(\alpha)$.

If moreover $P(\alpha)=N B(\alpha)$ then we conclude by Theorem 3.4 that $\varphi=\varphi_{*}$ on $X$ is a viscosity solution to the equation $\left(D M A_{v}^{1}\right)$ on $X$, hence $e^{\varphi}$ is continuous on $X$.

\section{Pluripotential tools}

4.1. Stability inequalities for big classes. The following result is the main stability inequality established in [GZ12]:

Theorem 4.1. Assume $\left(\theta+d d^{c} \varphi_{\mu}\right)^{n}=f_{\mu} \omega_{X}^{n},\left(\theta+d d^{c} \varphi_{\nu}\right)^{n}=f_{\nu} \omega_{X}^{n}$, where the densities $0 \leq f_{\mu}, f_{\nu}$ are in $L^{p}\left(\omega_{X}^{n}\right)$ for some $p>1$ and $\varphi_{\mu}, \varphi_{\nu} \in$ $P S H(X, \theta)$ are normalized by $\sup _{X} \varphi_{\mu}=\sup _{X} \varphi_{\nu}=0$. Then

$$
\left\|\varphi_{\mu}-\varphi_{\nu}\right\|_{L^{\infty}(X)} \leq M_{\tau}\left\|f_{\mu}-f_{\nu}\right\|_{L^{1}(X)}^{\tau}
$$

where $M_{\tau}>0$ only depends on upper bounds for the $L^{p}$ norms of $f_{\mu}, f_{\nu}$ and

$$
0<\tau<\frac{1}{2^{n}(n q+1)-1}, \quad \frac{1}{p}+\frac{1}{q}=1 .
$$

Corollary 4.2. Let $\alpha \in H^{1,1}(X, \mathbb{R})$ be a big cohomology class and assume $\varepsilon>0$ and $v \geq 0$ is a probability measure with $L^{p}$-density with respect to Lebesgue measure, where $p>1$. Then there exists a unique $\theta$ plurisubharmonic function $\varphi$ with minimal singularities which is a pluripotential solution of $\left(D M A_{v}^{\varepsilon}\right)$ on $X$

Moreover $\varphi$ is continuous on $\operatorname{Amp}(\alpha)$ and if $P(\alpha)=N B(\alpha)$ then $e^{\varphi}$ is also continuous on $X$.

Proof. The first part follows from [BEGZ10] and we get a unique pluripotential solution $\varphi$ with minimal singularities. Let $f$ denote the density of $v=f \omega_{X}^{n}$. We can approximate $f$ by continuous and positive densities by using convolutions, locally $f_{\delta}:=f \star \chi_{\delta}+\delta, \delta>0$. Theorem 3.5 insures that there exists a unique $\varphi_{\delta} \in P S H(X, \theta)$ solution to

$$
\left(\theta+d d^{c} \varphi_{\delta}\right)^{n}=e^{\varepsilon \varphi_{\delta}} f_{\delta} \omega_{X}^{n}
$$


which has minimal singularities and is continuous in $\operatorname{Amp}(\alpha)$. It follows moreover from [BEGZ10] Theorem 4.1 that the functions $\varphi_{\delta}-V_{\theta}$ are uniformly bounded as $\delta \rightarrow 0^{+}$.

The family $\left\{\varphi_{\delta}\right\}_{\delta>0}$ is compact in the $L^{1}$ topology hence we can extract a sequence $\left(\delta_{k}\right)_{k}$ such that $\varphi_{\delta_{k}}$ converges almost everywhere and $\sup _{X} \varphi_{\delta_{k}}$ converges. We can now apply the stability inequality (Theorem 4.1) to $\tilde{\varphi}_{\delta}:=\varphi_{\delta}-\sup _{X} \varphi_{\delta}$ to check that the functions $\tilde{\varphi}_{\delta_{k}}$ form a Cauchy sequence, so that the functions $\varphi_{\delta_{k}}$ form a Cauchy sequence too. The uniform limit $\phi=\lim \varphi_{\delta_{k}}$ has minimal singularities and satisfies $\left(\theta+d d^{c} \phi\right)^{n}=e^{\varepsilon \phi} f \omega_{X}^{n}$ in the pluripotential sense, hence coincides with $\varphi$.

\subsection{The flat setting.}

Theorem 4.3. Let $\alpha \in H^{1,1}(X, \mathbb{R})$ be a big cohomology class and assume $v \geq 0$ is a volume form with non-negative $L^{p}$-density with respect to Lebesgue measure such that $\int_{X} v=\operatorname{Vol}(\alpha)$ and $p>1$. Then there exists a unique $\theta$ plurisubharmonic function $\varphi$ with minimal singularities which is a pluripotential solution of $\left(D M A_{v}^{0}\right)$ on $X$ and such that $\int_{X} \varphi d v=0$.

Moreover $\varphi$ is continuous on $\operatorname{Amp}(\alpha)$ and if $P(\alpha)=N B(\alpha)$ then $e^{\varphi}$ is also continuous on $X$.

Proof. Fix $\varepsilon>0$ and let $\varphi_{\varepsilon}$ be the unique solution of $\left(D M A_{v}^{\varepsilon}\right)$ given by Corollary 4.2. It follows from [BEGZ10] that the functions $\varphi_{\varepsilon}-V_{\theta}$ are uniformly bounded, hence the argument of Corollary 4.2 enables to extract a Cauchy sequence whose limit $\psi$ is a solution of $\left(D M A_{v}^{0}\right)$ which satisfies

$$
\int \psi d v=\lim _{\varepsilon \rightarrow 0} \int\left[\frac{e^{\varepsilon \varphi_{\varepsilon}}-1}{\varepsilon}\right] d v=\int \varphi d v=0,
$$

hence $\varphi=\psi$ (by the uniqueness result proved in [BEGZ10]) has all required properties.

Corollary 4.4. The function $V_{\theta}$ is continuous if and only if $P(\alpha)=N B(\alpha)$.

Proof. Observe that if $e^{V_{\theta}}$ is continuous then $P(\alpha)=N B(\alpha)$, as points in $N B(\alpha) \backslash P(\alpha)$ correspond to points where $V_{\theta}$ is finite but not locally finite.

Conversely it follows from the work of Berman-Demailly BD12 that $V_{\theta}$ has locally bounded Laplacian in $\operatorname{Amp}(\alpha)$ and satisfies

$$
\left(\theta+d d^{c} V_{\theta}\right)^{n}=\mathbf{1}_{\left\{V_{\theta}=0\right\}} \theta^{n} \text { in } \operatorname{Amp}(\alpha) .
$$

Since $\theta^{n}$ is smooth and the density $\mathbf{1}_{\left\{V_{\theta}=0\right\}}$ is bounded, it follows from previous theorem that $V_{\theta}$ is continuous on $X$ if $P(\alpha)=N B(\alpha)$.

4.3. Conclusion. The proof of the main theorem proceeds now exactly as in that of Theorem 2.3. if $\varphi$ is a given $\theta$-psh function, we approximate it from above by a decreasing sequence of smooth functions $h_{j}$ and set

$$
\varphi_{j}:=P\left(h_{j}\right) \in P S H(X, \theta) .
$$


These functions have minimal singularities, decrease to $\varphi$ and solve the complex Monge-Ampère equation

$$
\left(\theta+d d^{c} \varphi_{j}\right)^{n}=\mathbf{1}_{\left\{\varphi_{j}=h_{j}\right\}}\left(\theta+d d^{c} h_{j}\right)^{n}=f_{j} \omega_{X}^{n},
$$

where $f_{j}$ is bounded. It follows therefore from Theorem 4.3 that $\varphi_{j}$ is continuous if $P(\alpha)=N B(\alpha)$.

Let us conclude by mentionning that we don't know any example of a compact Kähler manifold $X$ and a big cohomology class $\alpha \in H^{1,1}(X, \mathbb{R})$ such that $P(\alpha)$ does not coincide with $N B(\alpha)$.

\section{REFERENCES}

[BT82] Bedford, E. Taylor, B.A. A new capacity for plurisubharmonic functions. Acta Math. 149 (1982), 1-40.

[BD12] Berman, R., Demailly, J.-P.: Regularity of plurisubharmonic upper envelopes in big cohomology classes. Perspectives in analysis, geometry, and topology, 3966, Progr. Math., 296, Birkhäuser/Springer, New York, 2012.

[Bou04] Boucksom, S. Divisorial Zariski decompositions on compact complex manifolds. Ann. Sci. Ecole Norm. Sup. (4) 37 (2004), no. 1, 45-76.

[BEGZ10] Boucksom, S. Eyssidieux P. Guedj, V. Zeriahi, A. Monge-Ampère equations in arbitrary cohomology classes. Acta Mathematica 205 (2010), 199-262.

[Ceg83] Cegrell, U. Discontinuité de l'opérateur de Monge-Ampère complexe. C. R. Acad. Sci. Paris Sér. I Math. 296 (1983), no. 21, 869-871.

[CGZ13] Coman, D., Guedj, V., Zeriahi, A. Extension of plurisubharmonic functions with growth control. Journal für die reine und angewandte Mathematik 676 (2013), 33-49.

[CIL92] Crandall, M., Ishii, H. , Lions, P.L. User's guide to viscosity solutions of second order partial differential equations. Bull. Amer. Math. Soc. 27 (1992), 1-67.

[Dem92] Demailly, J.-P. Regularization of closed positive currents and intersection theory. J. Algebraic Geom. 1 (1992), no. 3, 361-409.

[DG09] Diller, J., Guedj, V. Regularity of dynamical Green's functions. Trans. Amer. Math. Soc. 361 (2009), no. 9, 4783-4805.

[EGZ09] Eyssidieux, P., Guedj, V., Zeriahi, A. Singular Kähler-Einstein metrics. J. Amer. Math. Soc. 22 (2009), 607-639.

[EGZ11] Eyssidieux, P., Guedj, V., Zeriahi, A. Viscosity solutions to degenerate complex Monge-Ampère equations. Comm. Pure and Applied Math.64 (2011), 1059-1094.

[GZ12] Guedj, V., Zeriahi, A. Stability of solutions to complex Monge-Ampère equations in big cohomology classes. Math. Res. Lett. 19 (2012), no. 05, 1025-1042.

[Kol98] Kolodziej, S. The complex Monge-Ampère equation. Acta Math. 180 (1998),69117.

[R68] Richberg, R. Stetige streng pseudokonvexe Funktionen. Math.Ann. 175 (1968),257286.

[Yau78] Yau, S.T. On the Ricci curvature of a compact Kähler manifold and the complex Monge-Ampère equation., Comm. Pure Appl. Math. 31, 339-441 (1978).

Université Joseph Fourier et Institut Universitaire de France, France

E-mail address: Philippe.Eyssidieux@ujf-grenoble.fr

Institut de Mathématiques de Toulouse et Institut Universitaire de France, Université Paul Sabatier, 118 route de Narbonne, F-31062 Toulouse Cedex 09,

E-mail address: vincent.guedj@math.univ-toulouse.fr 
Institut de Mathématiques de Toulouse,, Université Paul Sabatier, 118 route de Narbonne, F-31062 Toulouse Cedex 09,

E-mail address: zeriahi@math.univ-toulouse.fr 